\title{
A Simple Method for Constructing Small Cubic Graphs of Girths 14,15 , and 16
}

\author{
Geoffrey Exoo \\ Department of Mathematics and Computer Science \\ Indiana State University \\ Terre Haute, IN 47809
}

Submitted: September 20, 1996; Accepted: September 26, 1996

\begin{abstract}
A method for constructing cubic graphs with girths in the range 13 to 16 is described. The method is used to construct the smallest known cubic graphs for girths 14, 15 and 16 .
\end{abstract}

\section{Introduction}

We consider the problem of finding smallest regular graphs with given girth and degree: the cage problem. This problem has a prominent place in both Extremal and Algebraic Graph Theory. Biggs book on Algebraic Graph Theory [1] provides an introduction to this subject. Wong's survey article [3] gives a comprehensive picture of the state of the art in 1982. In this note, we shall be specifically interested in cubic (trivalent) cages and note that the current status of the problem can be obtained from Gordon Royle [2].

\section{The General Construction}

We describe a family of cubic graphs with girths in the stated range. We begin with a description of a type of generalized Petersen graph that does not quite achieve our girth objectives, and then consider adjustments to the construction that will increase the girth.

Let $T$ be a cubic tree (a tree in which each vertex has degree 1 or 3 ) on $t$ vertices. Then $t$ is even and $r=t / 2+1$ is the number of endvertices. Let $T_{1}, \ldots, T_{n}$ be $n$ copies of $T$. Suppose the 
vertices of $T_{i}$ are labeled $v_{i, 0}, \ldots, v_{i, t-1}$ so that vertices $v_{i, 0} \ldots v_{i, r-1}$ are the endvertices. Initially we require that the mapping from $T_{i}$ to $T_{j}$ given by $v_{i, k} \rightarrow v_{j, k}$ be an isomorphism, for all $\mathrm{i}$ and $\mathrm{j}$. In this case we can obtain a simple generalization of the Petersen graph by choosing $r$ positive integers $h_{1} \ldots h_{r}, h_{i}<n / 2$, and joining vertex $v_{i, k}$ to $v_{i+h_{i}, k}$. This gives a trivalent graph. It will be convenient to call the edges in one of the $T_{i}$ 's tree edges and the edges joining two of the trees chords. Note that for the Petersen graph we have $n=5, T=K_{2}, h_{1}=1$, and $h_{2}=2$.

Any tree with degree set $\{1,3\}$ has two endvertices at distance 2 . Therefore the graphs described in the previous paragraph have girth at most 12, since we can find a 12-cycle consisting of four pairs of tree edges (each pair joining two endvertices at distance 2) and four chords. Specifically, let $v_{i, x}$ and $v_{i, y}$ be endvertices adjacent to $v_{i, z}$ in $T_{i}$. Then the following vertices determine a 12-cycle: $v_{i, x}, v_{i, z}, v_{i, y}, v_{i+h_{y}, y}, v_{i+h_{y}, z}, v_{i+h_{y}, x}, v_{i+h_{y}+h_{x}, x}, v_{i+h_{y}+h_{x}, z}, v_{i+h_{y}+h_{x}, y}, v_{i+h_{x}, y}, v_{i+h_{x}, z}$, and $v_{i+h_{x}, x}$ (addition of subscripts in modulo $n$ ).

To achieve larger girth, we make a modification to the construction. First we drop the requirement that the mapping $v_{i, k} \rightarrow v_{j, k}$ be an isomorphism for all $i$ and $j$ and instead require that it be an isomorphism when $i-j$ is even. When $i-j$ is odd, we require only that it be a bijection that maps endvertices to endvertices. Note that parity considerations become important here, so we must have $n$ even.

\section{A Cubic Graph of Girth 14}

For example, let $T$ be the cubic tree on 6 vertices. For even $i$, we label it so that it's five edges are:

$$
\left(v_{i, 0}, v_{i, 4}\right),\left(v_{i, 1}, v_{i, 4}\right),\left(v_{i, 2}, v_{i, 5}\right),\left(v_{i, 3}, v_{i, 5}\right), \text { and }\left(v_{i, 4}, v_{i, 5}\right) .
$$

For odd $i$, we label so that the edges are:

$$
\left(v_{i, 0}, v_{i, 4}\right),\left(v_{i, 3}, v_{i, 4}\right),\left(v_{i, 1}, v_{i, 5}\right),\left(v_{i, 2}, v_{i, 5}\right), \text { and }\left(v_{i, 4}, v_{i, 5}\right) \text {. }
$$

Also, we let: $h_{0}=1, h_{1}=22, h_{2}=9$, and $h_{3}=34$. and finally, $n=82$. This gives us a trivalent graph on 492 vertices. It can be checked by computer that this graph has girth 14, and is the smallest known such graph [2].

\section{A Cubic Graph of Girth 15}

For our girth 15 construction we use a tree on 14 vertices which we label so that the edge list is as follows:

$$
\begin{gathered}
\left(v_{i, 0}, v_{i, 8}\right),\left(v_{i, 1}, v_{i, 8}\right),\left(v_{i, 2}, v_{i, 9}\right),\left(v_{i, 3}, v_{i, 9}\right),\left(v_{i, 4}, v_{i, 10}\right),\left(v_{i, 5}, v_{i, 10}\right),\left(v_{i, 6}, v_{i, 11}\right),\left(v_{i, 7}, v_{i, 11}\right) \\
\left(v_{i, 8}, v_{i, 12}\right),\left(v_{i, 9}, v_{i, 12}\right),\left(v_{i, 10}, v_{i, 13}\right),\left(v_{i, 11}, v_{i, 13}\right), \text { and }\left(v_{i, 12}, v_{i, 13}\right) .
\end{gathered}
$$


THE EleCtronic Journal of COMBinatorics 3 (1996), \#R30

For odd $i$, we label $T_{i}$ so that it's edges are:

$$
\begin{gathered}
\left(v_{i, 0}, v_{i, 8}\right),\left(v_{i, 6}, v_{i, 8}\right),\left(v_{i, 2}, v_{i, 9}\right),\left(v_{i, 4}, v_{i, 9}\right),\left(v_{i, 1}, v_{i, 10}\right),\left(v_{i, 5}, v_{i, 10}\right),\left(v_{i, 3}, v_{i, 11}\right),\left(v_{i, 7}, v_{i, 11}\right) \\
\left(v_{i, 8}, v_{i, 12}\right),\left(v_{i, 9}, v_{i, 12}\right),\left(v_{i, 10}, v_{i, 13}\right),\left(v_{i, 11}, v_{i, 13}\right), \text { and }\left(v_{i, 12}, v_{i, 13}\right) .
\end{gathered}
$$

And we let $h_{0}=1, h_{1}=11, h_{2}=9, h_{3}=19, h_{4}=7, h_{5}=37, h_{6}=17$, and $h_{7}=13$. If we also let $n=80$, we have a trivalent graph of order 1120 having girth 15, once again the smallest such graph known [2].

\section{A Cubic Graph of Girth 16}

For girth 16 we take $n=140$ and use the complete binary tree with radius two. For even $i$ we label $T_{i}$ so that its edges are:

$$
\begin{gathered}
\left(v_{i, 0}, v_{i, 6}\right),\left(v_{i, 1}, v_{i, 6}\right),\left(v_{i, 2}, v_{i, 7}\right),\left(v_{i, 3}, v_{i, 7}\right),\left(v_{i, 4}, v_{i, 8}\right),\left(v_{i, 5}, v_{i, 8}\right),\left(v_{i, 6}, v_{i, 9}\right),\left(v_{i, 7}, v_{i, 9}\right), \text { and } \\
\left(v_{i, 8}, v_{i, 9}\right) .
\end{gathered}
$$

Whereas for odd $i$ our labeling gives the edges:

$$
\begin{gathered}
\left(v_{i, 1}, v_{i, 6}\right),\left(v_{i, 2}, v_{i, 6}\right),\left(v_{i, 3}, v_{i, 7}\right),\left(v_{i, 4}, v_{i, 7}\right),\left(v_{i, 5}, v_{i, 8}\right),\left(v_{i, 0}, v_{i, 8}\right),\left(v_{i, 6}, v_{i, 9}\right),\left(v_{i, 7}, v_{i, 9}\right), \text { and } \\
\left(v_{i, 8}, v_{i, 9}\right) .
\end{gathered}
$$

Also we let $h_{0}=1, h_{1}=9, h_{2}=23, h_{3}=57, h_{4}=67$, and $h_{5}=43$., thereby producing a trivalent graph of order 1400 with girth 16, the smallest such graph yet discovered [2].

\section{A Final Note}

For a given $n$ and $t$, it is fairly easy to do an exhaustive search through all graphs of this type discussed above. So we were able to verify that these are the smallest such graphs with the stated girth values. In addition, other similar families were considered. We relaxed the requirement that

$T$ be a tree, and also considered cubic forests. And we tried replacing the single values of $h_{i}$ by sets of values (we tried sets of size two). Neither of these modifications produced better constructions.

\section{References}

[1] N.L. Biggs, Algebraic Graph Theory (2nd ed.), Cambridge University Press, 1993.

[2] G. Royle, Cubic Cages, 〈http://www.cs.uwa.edu.au/ gordon/cages/index.html $\rangle$, September, 1996 (Accessed: September 20, 1996).

[3] P.K. Wong. Cages - a survey, Journal of Graph Theory, 6, 1982, 1-22. 